\title{
Model Integration and Cyber Physical Systems: A Semantics Perspective
}

\author{
Janos Sztipanovits \\ Institute for Software Integrated Systems, Vanderbilt University, P.O. Box 1829 Sta. B. \\ Nashville, TN 37235, USA \\ janos.sztipanovits@vanderbilt.edu
}

\begin{abstract}
Recent attention to Cyber Physical Systems (CPS) is driven by the need for deeper integration of design disciplines that dominate physical and computational domains. Consequently, heterogeneity is the norm as well as the main challenge in CPS design: components and systems are modeled using multiple physical, logical, functional and non-functional modeling aspects. The scope of relevant design domains includes (1) physical domains, such as structure, mechanical dynamics, thermal, propulsion, fluid, electrical, acoustics/vibration and (2) computational/networking domains, such as system control, sensors, health management, mission management, communication. However, the practice of multi-modeling - using established domain-specific modeling languages and tools independently in the design process - is insufficient. Modeling and analyzing cross-domain interactions among physical and computational/networking domains and understanding the effects of heterogeneous abstraction layers in the design flow are fundamental part of CPS design theories. I will cast this challenge as a model integration problem and discuss solutions for capturing interdependencies across the modeling domains using constructs for meta-model composition and integration.
\end{abstract}

